\title{
GYNOSTEMMA (CUCURBITACEAE) IN THAILAND AND MALESIA
}

\author{
W.J.J.O. DE WILDE \& B.E.E. DUYFJES \\ Nationaal Herbarium Nederland, Universiteit Leiden branch, P.O. Box 9514, \\ 2300 RA Leiden, The Netherlands; e-mail: dewilde@nhn.leidenuniv.nl
}

\begin{abstract}
SUMMARY
The genus Gynostemma is represented in Thailand and Malesia by four species of which the extremely variable G. pentaphyllum is widespread covering the whole area. Seven forms are recognized in G. pentaphyllum, of which three are new: forma fasciculare W.J. de Wilde \& Duyfjes, forma grandiflorum W.J. de Wilde \& Duyfjes, forma knemandrum W.J. de Wilde \& Duyfjes, and three are new combinations: forma dasycarpum (C.Y. Wu) W.J. de Wilde \& Duyfjes, forma pubescens (Gagnep.) W.J. de Wilde \& Duyfjes, and forma simplicifolium (Blume) W.J. de Wilde \& Duyfjes. Two new species, both endemic to New Guinea, are described: G. intermedium W.J. de Wilde \& Duyfjes and G. papuanum W.J. de Wilde \& Duyfjes.
\end{abstract}

Key words: Cucurbitaceae, Gynostemma, South East Asia.

\section{INTRODUCTION}

Gynostemma Blume is a genus of mostly small climbers comprising about 10 species. The largest diversity of species is found in China (Wu \& Chen, 1983; Chen, 1986, $1995 \mathrm{a}, \mathrm{b})$. Usually the genus can be easily recognized by foliolate leaves, minute male flowers of which the staminal filaments are fused into a column and the anthers arranged around a small stipitate disc, and small pea-like fruits. The Gynostemma species in Thailand and Malesia seem closely related and closely resemble each other. Only G. microspermum, from northern Thailand (and southern China) significantly differs in its fruit, which is capsular and dry, and not berry-like. Therefore it was placed with some other species having similar fruits in a separate genus, Trirostellum (Wang \& Xie, 1981), or as Gynostemma subg. Trirostellum (Wu \& Chen, 1983). Recently in China, in this latter subgenus, some species with 5 styles instead of the usually 3 have been recognized as a distinct section, sect. Pentastylos (Chen, 1995b). However, species with 5 styles in subg. Gynostemma occur in New Guinea as well, but we are not creating a separate section for these. Two species of Gynostemma are accepted for Thailand and three for the Malesian area, of which two are new and here described: G. intermedium and G. papuanum. The most widespread and unbelievably variable species is G. pentaphyllum, occurring in both Thailand and Malesia. A large part of its variation is rather arbitrarily described by recognizing a number of local or regional forms. 


\section{GYNOSTEMMA}

Gynostemma Blume (1825) 23; Hook.f. in Benth. \& Hook.f. (1867) 839; C.B. Clarke (1879) 633; Cogn. (1881) 912; E.G.O. Müll. \& Pax (1889) 37; Gagnep. (1921) 1079; Chakrav. (1959) 187; Backer (1964) 305; Ohwi (1965) 848; Keraudren (1975) 24; C. Jeffrey (1980) 10. - Pestalozzia Zoll. \& Moritzi in Moritzi (1846) 31 (nom. superfl.). - Type species: Gynostemma pedatum Blume (Java).

Trirostellum Z.P. Wang \& Q.Z. Xie (1981) 481 - Gynostemma Blume subg. Trirostellum (Z.P. Wang \& Q.Z. Xie) C.Y. Wu \& S.K. Chen (1983) 364; S.K. Chen (1986) 273; (1995b) 405. - Type species: Trirostellum yixingensis Z.P. Wang \& Q.Z. Xie.

Enkylia Griff. (1854) 645. - Lectotype species (here chosen): Enkylia trigyna Griff.

Small or medium climbers; main branches subherbaceous or soft woody; roots tuberous or not; dioecious or monoecious; twigs slender, glabrous or finely hairy. Probract absent. Tendrils terminally 2-branched. Leaves: blade simple, not lobed or subpedately 3- or 5- (or 7-)foliolate, green on drying; petiole long; leaflets petioluled, margin dentate; cystoliths not obvious. Flowers small, 2-5(-8) mm diam., rotate, greenish white or white; buds globose; sepals 5, free; petals 5, free, imbricate in bud, long-triangular, sometimes \pm erose towards apex; disc inconspicuous. Male inflorescences: paniculate, 2-4-times branched, few- or many-flowered, the ultimate branches fine with few or many \pm clustered flowers in minute short racemes; bracts small, linear, $0.5-1(-2) \mathrm{mm}$ long, glabrous or finely hairy. Male flowers: pedicel short, articulate, basal part persistent; receptacle narrow, flat or shallowly hollowed; sepals c. $1 \mathrm{~mm}$ long; petals (long-) triangular or narrowly elliptic, apex acute or (long-)acuminate; stamens: filaments united into a short staminal column, at apex with 5 (sub) sessile or short-stiped anthers in a ring (synandrium), anthers small, 1-thecous, short-ellipsoid, opening with a vertical slit, extrorse. Female inflorescences as in male but less ramified and smaller. Female flowers: similar to but somewhat larger than male flowers; pedicel short (or long); ovary subglobose, largely inferior, the inferior part not narrowed below the perianth, (2- or) 3- or 5-locular, each locule with 2 hanging ovules; sepals and petals inserted along broad, slightly convex, apex of ovary; styles ( 2 or) 3 or 5 , erect, \pm contiguous, glabrous, each at apex with a 2-branched stigma; staminodes sometimes present(?). Fruits: (sub)globose, less than $10 \mathrm{~mm}$ diam., with subpersistent perianth and styles (the latter becoming spaced and the perianth-scars leaving a thin line around the fruit), either a dry berry, not dehiscent (subg. Gynostemma) or \pm capsular, opening with 3 valves between the styles (subg. Trirostellum). Seeds: few, 1-5, not much compressed, ovoid or subtriangular in outline, faces verrucose, margin narrow or broad, \pm square.

A genus of about ten species, occurring in Sri Lanka and in S and NE India, ranging through China east to Japan, and into Malesia east to New Guinea. Two species in Thailand, three species in Malesia.

Notes -1 . Although the androecium is characteristic for the genus, two species of the genus Neoalsomitra, namely $N$. angustipetala (Craib) Hutch. and N. hederifolia (Decne.) W.J. de Wilde \& Duyfjes, have a similar androecium (De Wilde \& Duyfjes, 2003).

2. The basic chromosome number of the two subgenera is $x=11$ (Gao et al., 1995). Various levels of polyploidy were found by the latter authors in several species, and sometimes even in one population of a form. 


\section{KEY TO THE SPECIES}

1a. Fruit capsular, 3-4 mm diam., opening at apex with 3 valves. Seeds c. $2 \mathrm{~mm}$ diameter. Inflorescences short, condensed. [Subg. Trirostellum]. - N Thailand (and China $\ldots \ldots \ldots \ldots \ldots \ldots \ldots \ldots \ldots \ldots \ldots \ldots \ldots \ldots$ 4. G. microspermum

b. Fruit a dry berry, 5-10(-12) mm diam., indehiscent. Seeds $2.5-4 \mathrm{~mm}$ diameter. Inflorescences short or long, usually lax. [Subg. Gynostemma] . . . . . . . . 2

2a. Monoecious. Inflorescences few-flowered. Plant delicate. - Papua New Guinea;

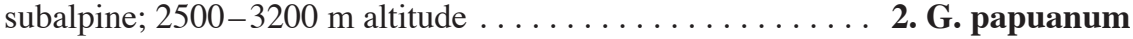

b. Dioecious. Inflorescences many-flowered. Plant stouter . . . . . . . . . . 3

3a. Styles 5. - Eastern Papua New Guinea; montane; 1000-2500 m altitude . . . . . $\ldots \ldots \ldots \ldots \ldots \ldots \ldots \ldots \ldots \ldots \ldots \ldots \ldots \ldots \ldots \ldots \ldots$. G. intermedium

b. Styles (2 or) 3. - Widespread, lowland and montane areas; in New Guinea $200-1000(-1800) \mathrm{m} \ldots \ldots \ldots \ldots \ldots$. . . . . . . pentaphyllum (with 7 forms)

\section{Subgenus GYNOSTEMMA}

Fruit a dry berry, not opening with valves.

1. Gynostemma intermedium W.J. de Wilde \& Duyfjes, spec. nov. - Fig. 1a, b

A Gynostemmatis consubgeneribus novoguineensibus floribus foemineis stylis 5, fructu maiore 6-12 mm diam. differt. - Typus: Pullen 422, female flowers (holo L; iso CANB), Papua New Guinea, Eastern Highlands.

Gynostemma pentaphyllum non (Thunb.) Makino: P. Royen (1982) 2008, p.p., f. 613A.

Small climber or creeper, 1-2 m long, root not known; leafy stem 1-1.5 mm thick, sparsely hairy, glabrescent; dioecious. Leaves: blade membranous, (3 - or) 5 - or 7 foliolate, ovate or circular in outline, $3-6 \mathrm{~cm}$ diam.; petiole $2-3.5 \mathrm{~cm}$ long; leaflets up to 5 by $2(-2.5) \mathrm{cm}$, margin (shallowly) coarsely serrate, middle leaflet largest, with 5-7 pairs of rather faint lateral nerves, upper surface sparsely hairy mainly on nerves, lower surface subglabrous; petiolule $0.5-1.5 \mathrm{~cm}$ long. Male inflorescences: many-flowered, paniculate, ( 2 or) 3 times branched, $10-15$ by $5-10 \mathrm{~cm}$, ultimately with up to 5 flowers in fascicles or short racemes up to $15 \mathrm{~mm}$ long; peduncle c. 3 $\mathrm{cm}$ long, finely pubescent; bracts minute, less than $1 \mathrm{~mm}$ long. Male flowers: pedicel 2-3 mm long, (sub)glabrous, articulation indistinct, at c. 1/3 from the apex; perianth glabrous, 4.5(-5) mm diam.; receptacle shallow, 0.6-0.9 mm wide; sepals ovatenarrowly elliptic, c. 1 by $0.5 \mathrm{~mm}$; petals triangular-ovate, c. 2 by $1(-1.5) \mathrm{mm}$, entire, with narrowed apiculate apex; staminal column (0.3-) $0.5 \mathrm{~mm}$ long, synandrium c. $0.5 \mathrm{~mm}$ diam., anthers subsessile, subglobose, c. $0.2 \mathrm{~mm}$ diameter. Female inflorescences as in male, paniculate, rather many-flowered, $1(-3)$ times branched, 5-10 cm long; peduncle 1.5-3 cm long, (sparsely) hairy. Female flowers: pedicel 3-4 mm long, articulation not seen; ovary (hemi-)globose, c. $1.5 \mathrm{~mm}$ diam., (sparsely) hairy or glabrous; perianth c. $4 \mathrm{~mm}$ diam.; styles 5, each c. $1 \mathrm{~mm}$ long. Fruits: few or up to 20 per infructescence, globose, 6-10(-12) mm diam., with 5 style-remnants and 5 (not opening) valvesutures, glabrous, pale brown when dry; fruiting pedicel c. $5 \mathrm{~mm}$ long. Seeds: 3 or 4 per berry, rounded or subtriangular, c. 4 by 3.5 by $2 \mathrm{~mm}$, with \pm flattish apex, pale brown, coarsely sparsely warted or irregularly ridged, margin narrow. 


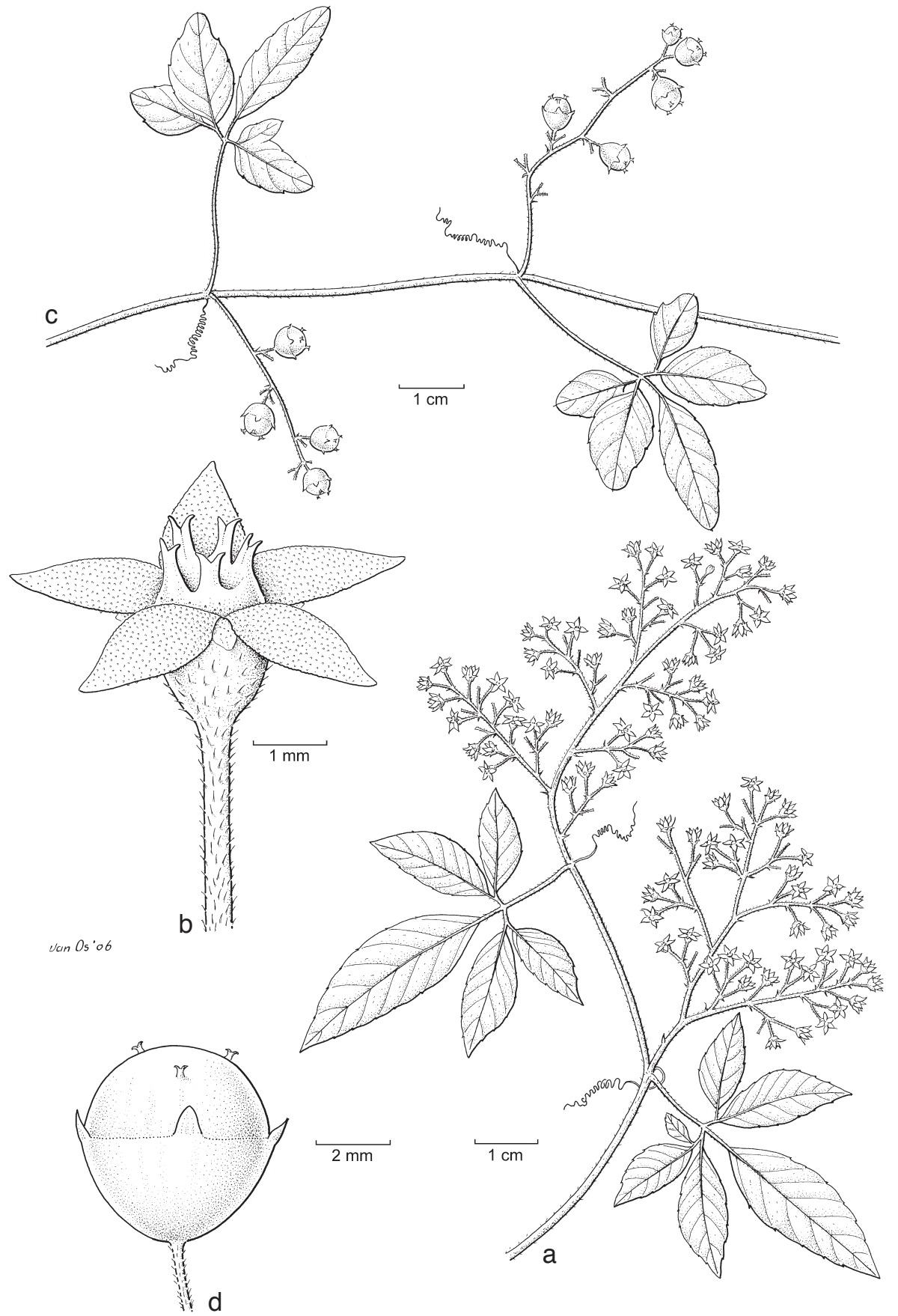

Fig. 1. a, b: Gynostemma intermedium W.J. de Wilde \& Duyfjes. a. Habit of shoot with female inflorescences; b. female flower. - c, d: Gynostemma papuanum W.J. de Wilde \& Duyfjes. c. Stem section with fruit; d. fruit (a, b: Pullen 422 (type); c, d: Stevens LAE 55693). 
Distribution - Eastern Papua New Guinea: Madang, Eastern Highlands, Morobe; known from 6 collections.

Habitat \& Ecology - Climber on tree ferns; along stream sides and gullies in mountain forest; at 1500 up to $2500 \mathrm{~m}$ altitude. Flowering and fruiting throughout the year.

Field-notes - Flowers green. Fruits green, shiny or dark purple with a sharp biting taste.

Note - Gynostemma intermedium occupies in East New Guinea the altitudinal montane zone between G. pentaphyllum of the lowlands and G. papuanum of the subalpine zone.

Specimens examined:

Coode NGF 32683, Floyd \& Womersley 6779, Pullen 422, Sayers NGF 19787, Van Royen \& Millar NGF 17541, Womersley \& Sleumer NGF 13999.

2. Gynostemma papuanum W.J. de Wilde \& Duyfjes, spec. nov. - Fig. 1c, d

A Gynostemmatis consubgeneribus habitu monoecio, inflorescentiis masculis paucifloris, floribus foemineis stylis 3 (vel 4), seminibus c. 2.5 mm diam. differt. - Typus: Vink 17364 (holo L), Papua New Guinea, Southern Highlands.

Gynostemma pentaphyllum non (Thunb.) Makino: P. Royen (1982) 2008, p.p., f. 613: B-D.

Small climber, 0.5-1 m long, root not known; leafy stem less than $1 \mathrm{~mm}$ thick, subglabrous or sparsely hairy; monoecious. Leaves: blade filmy or membranous, (3- or) 5 -foliolate, ovate in outline, $3-6$ by $2.5-4.5 \mathrm{~cm}$; petiole $1.5-2.5 \mathrm{~cm}$ long; the middle leaflet much longer than the lateral ones, with c. 5 pairs of faint lateral nerves, margin shallowly crenate-dentate, both surfaces subglabrous or sparsely hairy; petiolule $0.2-0.4 \mathrm{~cm}$ long. Inflorescences few-flowered, 1-2(-3) cm long, consisting of 1 (or 2) female flowers and later developing 1-3(-5) male flowers, inflorescence solitary or \pm fascicled, sometimes only female or only male flowers present in one inflorescence, sometimes very short peduncled inflorescences aggregated in raceme-like short shoots; branches and pedicels hairy; peduncle $0.5-2 \mathrm{~cm}$ long; bracts narrow, acute, $1 \mathrm{~mm}$ long or less. Male flowers: pedicel 2-5 mm long, glabrous or hairy, articulate at c. 1/3 from the apex; perianth glabrous, c. $3 \mathrm{~mm}$ diam.; receptacle shallow, less than $0.5 \mathrm{~mm}$ wide; sepals ovate-narrowly elliptic, $0.7(-1) \mathrm{mm}$ long; petals ovate-narrowly elliptic, c. $1.5 \mathrm{~mm}$ long, entire or somewhat incised, long-acuminate in upper $1 / 3$; staminal column c. $0.3 \mathrm{~mm}$ long, synandrium $0.3(-0.4) \mathrm{mm}$ diam., anthers sessile, subglobose, c. $0.2 \mathrm{~mm}$ diameter. Female flowers: pedicel $2-5 \mathrm{~mm}$ long, articulate at apex; ovary (sub)hemi-globose, c. $0.6 \mathrm{~mm}$ diam., glabrous; perianth $2.5-3 \mathrm{~mm}$ diam.; sepals c. $0.5 \mathrm{~mm}$ long; petals ovate to narrowly elliptic, 1(-1.5) $\mathrm{mm}$ long, acuminate; styles 3 (rarely 4), each c. $1 \mathrm{~mm}$ long. Fruit: solitary, globose, 5-7 mm diam., pale brownyellow when dry (light green when fresh), with persistent sepals and styles and with 3 (not opening) valve-sutures; fruiting pedicel 4-6 mm long. Seeds: 2 or 3 per berry, \pm triangular-ovate, $2.5-3$ by $2-2.5$ by $1 \mathrm{~mm}$, (pale) brown, faces irregularly low-warty, margin narrow.

Distribution - Papua New Guinea: West Sepik, East Sepik, Western Highlands (Kubor Range), Southern Highlands (Mt Giluwe), Chimbu, Milne Bay (Mt Suckling); known from 13 collections. 
Habitat \& Ecology - Open places in low forest, mossy forest, gullies and low scrub, grasslands, alpine shrubbery and Nothofagus forest; also on limestone; at (1600-)2100-3500 m altitude. Flowering and fruiting throughout the year.

\section{Gynostemma pentaphyllum (Thunb.) Makino}

Gynostemma pentaphyllum (Thunb.) Makino (1902) 179; Backer (1964) 306; Ohwi (1965) 848; Keraudren (1975) 25, pl. 5: 1-7; C. Jeffrey (1980) 10; P.H. Hô (1991) 727, f. 2017. - Vitis pentaphylla Thunb. (1784) 105. - Type: Thunberg 5858 (UPS, not seen), Japan.

Gynostemma pedatum Blume (1825) 23; C.B. Clarke (1879) 633 ('pedata'); Cogn. (1881) 913; Gagnep. (1921) 1080; Craib (1931) 766; Chakrav. (1959) 188. - Pestalozzia pedata Zoll. \& Moritzi in Moritzi (1846) 31. - Zanonia pedata (Blume) Miq. (1856) 683. - Lectotype (here chosen): Blume 1429 (holo L, barcode L0588327; iso L (6 sheets)), Java, Tjanjor \& Krawang.

Gynostemma simplicifolium Blume (1825) 24 ('simplicifolia'); Cogn. (1881) 915; Chakrav. (1959) 193; Backer (1964) 306; F.C. How \& C.C. Chang (1964) 484; C.Y. Wu \& S.K. Chen (1983) 359.

- Lectotype (here chosen): Blume 1493 (holo L, barcode L0588361; iso L), Java, Mt Krawang.

Zanonia cissoides Wall. (1831) 28. - Alsomitra cissoides (Wall.) M. Roem. (1846) 118. - Type: Wallich 3726 (K, K-W), Nepal.

Zanonia laxa Wall. (1831) 29. - Alsomitra laxa (Wall.) M. Roem. (1846) 118. - Pestalozzia laxa (Wall.) Thwaites (1859) 124. - Gynostemma laxum (Wall.) Cogn. (1881) 914; Craib (1931) 766; Merr. (1938) 69; Chakrav. (1959) 190; Keraudren (1975) 26; P.H. Hô (1991) 726, f. 2016. - Type: Wallich 3727 (K, K-W), India, Silhet.

Zanonia wightiana Arn. (1836) 356b. - Gynostemma wightianum (Arn.) Hook.f. in Benth. \& Hook.f. (1867) 839. - Type: Walker? s.n. (holo E, in herb. Arnott), Ceylon.

Enkylia digyna Griff. (1854) 645, pl. 4: 1-8. - Type not indicated, lectotype not chosen, India, Khasia.

Enkylia trigyna Griff. (1854) 646, pl. 4: 9-15. - Type: not indicated, lectotype not chosen, India, Himalaya.

Vitis martini H. Lév. \& Vaniot (1905) 41. - Type (Lauener, 1972): Martin \& Bodinier 1791 (holo E), China, Kweichow, near Gan-pin.

Vitis mairei H. Lév. (1912b) 299, non H. Lév. (1909) 338. - Type (Lauener, 1972): Maire s.n, IX1911 (holo E), China, Yunnan, grottes de Pan-long-se.

Vitis quelpaertensis H. Lév. (1912a) 351. - Type (Lauener, 1972): Taquet 2745 (holo E; iso K), Korea, Quelpaert.

Gynostemma winkleri Cogn. in H. Winkl. (1912) 118. - Type: Winkler 2757 (B†; iso K, L), Indonesia, Borneo.

Gynostemma siamicum Craib (1918) 362; (1931) 767 ('siamica'); Merr. (1938) 70. - Lectotype (here chosen): Kerr 1434 (holo E; iso E, K), Thailand, Doi Sutep.

Gynostemma crenulatum Ridl. (1920) 93. - Type: Kloss 6901 (holo K), Peninsular Thailand.

Gomphogyne alleizettei Gagnep. (1921) 1086. - Type: Alleizette s.n. (holo P; iso P), Vietnam, Tonkin.

Gynostemma pubescens (Gagnep.) C.Y. Wu in C.Y. Wu \& S.K. Chen (1983) 362. - Gynostemma pedata Blume var. pubescens Gagnep. (1921) 1082. - Type: Thorel 3468 (holo P), Laos.

Gynostemma dasycarpum (C.Y. Wu in C.Y. Wu \& S.K. Chen) S.K. Chen (1995b) 406. - Type: $L i$ 534 (KUN, not seen), China, Yunnan.

Herbaceous or subwoody climber, 2-8 $\mathrm{m}$ long, root perennial but not(?) tuberous, main stem to $1 \mathrm{~cm}$ thick, bark fissured, grey; leafy stem slender, 1(-2) $\mathrm{mm}$ thick, glabrous or hairy, hairs grey or rarely brown. Leaves: blade simple or foliolate, subcircular (or ovate) in outline, 4-10(-17) cm diam.; petiole 3-6 cm long; leaflets $3-5(-7)$, ovate to narrowly elliptic, $3-10$ by $1-5 \mathrm{~cm}$, outer leaflets smallest, 3-6(-7)-pinninerved, nerves faint or conspicuous [distinct?], margin (remotely) finely (or coarsely) dentate, upper surface variously (appressed) hairy or subglabrous (except on the veins), lower 
surface (sub)glabrous; petiolule $2-7 \mathrm{~cm}$ long. Male inflorescences: many-flowered, small or large, raceme-like or 2-4-branched, broadly paniculate, $5-15(-30) \mathrm{cm}$ long, lateral branches to $10 \mathrm{~cm}$ long, hairy or subglabrous, ultimately with (1 or) $2-5$ flowers in fascicles (or in condensed racemes), finely hairy or subglabrous; peduncle 1-7 $\mathrm{cm}$ long; bracts 1-2(-5) mm long. Male flowers: pedicel 1-3(-7) mm long, articulate in the middle, the basal part persistent after flowering; perianth $(2.5-) 3-5(-8) \mathrm{mm}$ diam.; receptacle shallowly hollowed, c. $0.5 \mathrm{~mm}$ wide; sepals narrowly elliptic-linear, $(0.5-) 1 \mathrm{~mm}$ long; petals triangular, (narrowly) elliptic, $1-2(-3.5)$ by $0.5(-1) \mathrm{mm}$, apex acuminate or long-acuminate, adaxially usually \pm hairy or papillose; staminal column $0.5(-1) \mathrm{mm}$ long, synandrium $0.5(-1) \mathrm{mm}$ diam., anthers subglobose, sessile or very short-stalked, c. $0.2 \mathrm{~mm}$ diameter. Female inflorescences: as male inflorescences, but smaller. Female flowers: pedicel 2-4 mm long, subapically articulate; perianth 2-4 $\mathrm{mm}$ diam.; ovary subglobose, c. $1 \mathrm{~mm}$ diam., glabrous or sparsely hairy; styles (2 or) 3 , each $0.5-1 \mathrm{~mm}$ long. Fruits: globose, 5-8 $\mathrm{mm}$ diam., glabrous or (sparsely) hairy, green, pale yellow or blackish when ripe; fruiting pedicel $2-5 \mathrm{~mm}$ long. Seeds: usually 2 , subcircular, triangular or cordate in outline, 3-4 mm diam., little or moderately compressed, faces irregularly verrucose or wrinkled, margin broad or narrow.

Distribution - Widespread, as the genus; in India and Sri Lanka, east through China, to Japan and Korea, southeast through Indochina and Malesia to New Guinea. There are no records from Cambodia.

Habitat \& Ecology - In a variety of habitats: damp or not too dry places in scrub and forest, often near water courses, also on limestone; at 200-2400 m altitude; possible also at sea level in the lowlands. Flowering and fruiting throughout the year, but less frequent in April and May.

Note - Gynostemma pentaphyllum is very variable, comprising various not sharply demarcated local forms or ecotypes. Leaves can be either simple or very markedly 3 - or 5- (or 7-)foliolate. A form with strongly hairy leaves from Myanmar (also occurring in Thailand) was described as G. burmanicum (Chakravarty, 1946, invalid name), another hairy form from Indochina (Gagnepain, 1921) as G. pedatum var. pubescens. Plants with a sparsely hairy ovary and fruit have been found in Thailand. An obvious variation with (mostly) simple leaves is kept separate as forma simplicifolium. It is widespread and was originally described as a species from W Java, where it most likely originated locally. Similar plants, obviously, independently, locally developed, occur in Sabah (Kinabalu area), in the mountains of Peninsular Malaysia, and also in India and China. Plants with a sparsely hairy ovary have been found in Thailand, but similar plants with a hairy ovary and fruit have been recognized as distinct species: in Borneo (G. winkleri) and in China (G. dasycarpum), both are here united and reduced to the status of a form.

To acknowledge some of the variation, we recognize the following 7 forms:

a. forma dasycarpum (C.Y. Wu in C.Y. Wu \& S.K. Chen) W.J. de Wilde \& Duyfjes, stat. nov. - Plate 1c

Gynostemma pentaphyllum (Thunb.) Makino var. dasycarpum C.Y. Wu in C.Y. Wu \& S.K. Chen (1983) 362. - Gynostemma dasycarpum (C.Y. Wu in C.Y. Wu \& S.K. Chen) S.K. Chen (1995b) 406. - Type: Li 534 (KUN, not seen), China, Yunnan. 
Gynostemma winkleri Cogn. in H. Winkl. (1912) 118. - Type: Winkler 2757 (B†; iso K, L), Indonesia, Borneo.

Plant as forma pentaphyllum, but fruits hairy.

Distribution - All over Borneo, sporadically in Thailand.

Habitat \& Ecology - Primary forest, shaded and open places, damp places, riverbanks, and hillsides; often on limestone, also on basalt bedrock; mostly in the lowlands up to $1650 \mathrm{~m}$ altitude.

Notes - 1. Plants with hairy fruits are described as Enkylia digyna Griff. (1854) 645, from India.

2. Gynostemma yixingense (Z.P. Wang \& Q.Z. Xie) C.Y. Wu \& S.K. Chen var. trichocarpum J.N. Ding (1990: 71), a taxon with hairy fruit, is not a synonym because it pertains to a species of section Trirostellum with capsular fruits.

\section{b. forma fasciculare W.J. de Wilde \& Duyfjes, forma nov.}

A formis ceteris in pedicellis masculis persistentiter fasciculatis comparative longis plus quam 4 (vel 5) mm versus 1-2 mm longis differt. - Typus: Johansson, Nybom \& Riebe 384 (holo BO; iso L, S), Indonesia, Central Sulawesi.

Plant as forma pentaphyllum, but male flowers long-pedicelled, pedicels 4(-5) $\mathrm{mm}$ long, fascicled.

Distribution - Endemic to Central Sulawesi.

Habitat \& Ecology - Primary and disturbed primary forest on alluvial soil; from sea level up to $2200 \mathrm{~m}$ altitude.

Note - In Sulawesi the typical form pentaphyllum seems absent.

\section{c. forma grandiflorum W.J. de Wilde \& Duyfjes, forma nov.}

A forma typica in floribus masculis maioribus petalis $3.5 \mathrm{~mm}$ longis differt. - Typus: Maxwell 94-1001 (holo BKF; iso L), Thailand, Doi Inthanon NP.

Plant as forma pentaphyllum, but male flowers large, petals $3.5 \mathrm{~mm}$ long.

Distribution - This form is a local endemic of Thailand (Mt Inthanon).

Habitat \& Ecology - In Sphagnum bog just below the summit, elfin forest, primary evergreen hillside forest; on granite bedrock; at 700-2600 m altitude.

Note - The typical form pentaphyllum is also common in the summit region.

\section{d. forma knemandrum W.J. de Wilde \& Duyfjes, forma nov.}

Forma floribus masculis comparative minutis c. $2.5 \mathrm{~mm}$ diam., antheris in synandrio breviter stipitatis nec sessilibus. - Typus: Garrett 190 (holo L; iso E, K, L), Thailand, Chiang Rai.

Plant as forma pentaphyllum, but male flowers small, perianth c. $2.5 \mathrm{~mm}$ diam.; anthers in the synandrium shortly stipitate.

Distribution - Endemic to northern Thailand.

Habitat \& Ecology - Shaded in mixed evergreen and deciduous forest and on rugged limestone cliffs; only found on limestone; at 400-450 m altitude. 
e. forma pentaphyllum - Fig. 2b-f, 3; Plate 1b, d

For references see under the species.

Plant as the species, not including the forms here described as new. The leaves of the typical form are 3- or 5- (or 7-)foliolate. It is widespread in the lowlands up to 1500(-2000) $\mathrm{m}$ altitude. The form is rare in Borneo, where it is replaced by forma dasycarpum.

Uses - The form is cultivated for its medicinal properties. Tea prepared from the leaves, for instance, is used for reduction of blood pressure.

f. forma pubescens (Gagnep.) W.J. de Wilde \& Duyfjes, stat. nov.

Gynostemma pedatum Blume var. pubescens Gagnep. (1921) 1082. - Gynostemma pubescens (Gagnep.) C.Y. Wu in C.Y. Wu \& S.K. Chen (1983) 362. - Type: Thorel 3468 (holo P), Laos.

Gynostemma burmanicum Chakrav. (1946) 86; (1959) 190 ('burmanica'), not validly published, without Latin description.

Gynostemma burmanica Chakrav. var. molle C.Y. Wu in C.Y. Wu \& S.K. Chen (1983) 360, invalid name. - Voucher specimen: Wang 81064 (KUN, not seen), China, Yunnan.

Plant as forma pentaphyllum, but wholly, including both leaf blade surfaces, (densely) hairy.

Distribution - India, Myanmar, China, south-western and northern Thailand, Laos, Vietnam.

Habitat \& Ecology - Shaded in primary and disturbed forest, often along small streams; granite bedrock, also on limestone; at 600-1500 m altitude.

Field-note - Plants yellowish green.

Note - In G. burmanicum (nom. inval.) the hairs were described as red-brown.

g. forma simplicifolium (Blume) W.J. de Wilde \& Duyfjes, stat. nov. -

Fig. 2a; Plate 1a

Gynostemma simplicifolium Blume (1825) 24 ('simplicifolia'); Cogn. (1881) 915; Chakrav. (1959) 193; Backer (1964) 306; F.C. How \& C.C. Chang (1964) 484; C.Y. Wu \& S.K. Chen (1983) 359.

- Lectotype (here chosen): Blume 1493 (holo L, barcode L0588361; iso L), Java, Mt Krawang.

Plant as forma pentaphyllum, but leaves simple, occasionally with some 2 (or 3 ) foliolate leaves among the simple leaves.

Distribution - India, Myanmar, China, Peninsular Malaysia (Pahang), Borneo (Sarawak, Sabah), Java, Philippines (Basilan).

Habitat \& Ecology - Damp and shaded places in primary and old secondary forest, along roadsides in logged forest and river beds; yellow sandy and reddish clay lateritic soil, also on limestone; at 100-1600 m altitude.

Note - Simple-leaved (not foliolate) forms have been found almost all over the area of the species. Plants from the type locality in W Java have a relatively long petiole, almost as long as the rather narrow leaf blade. In simple-leaved forms from other localities the petiole is generally shorter. 


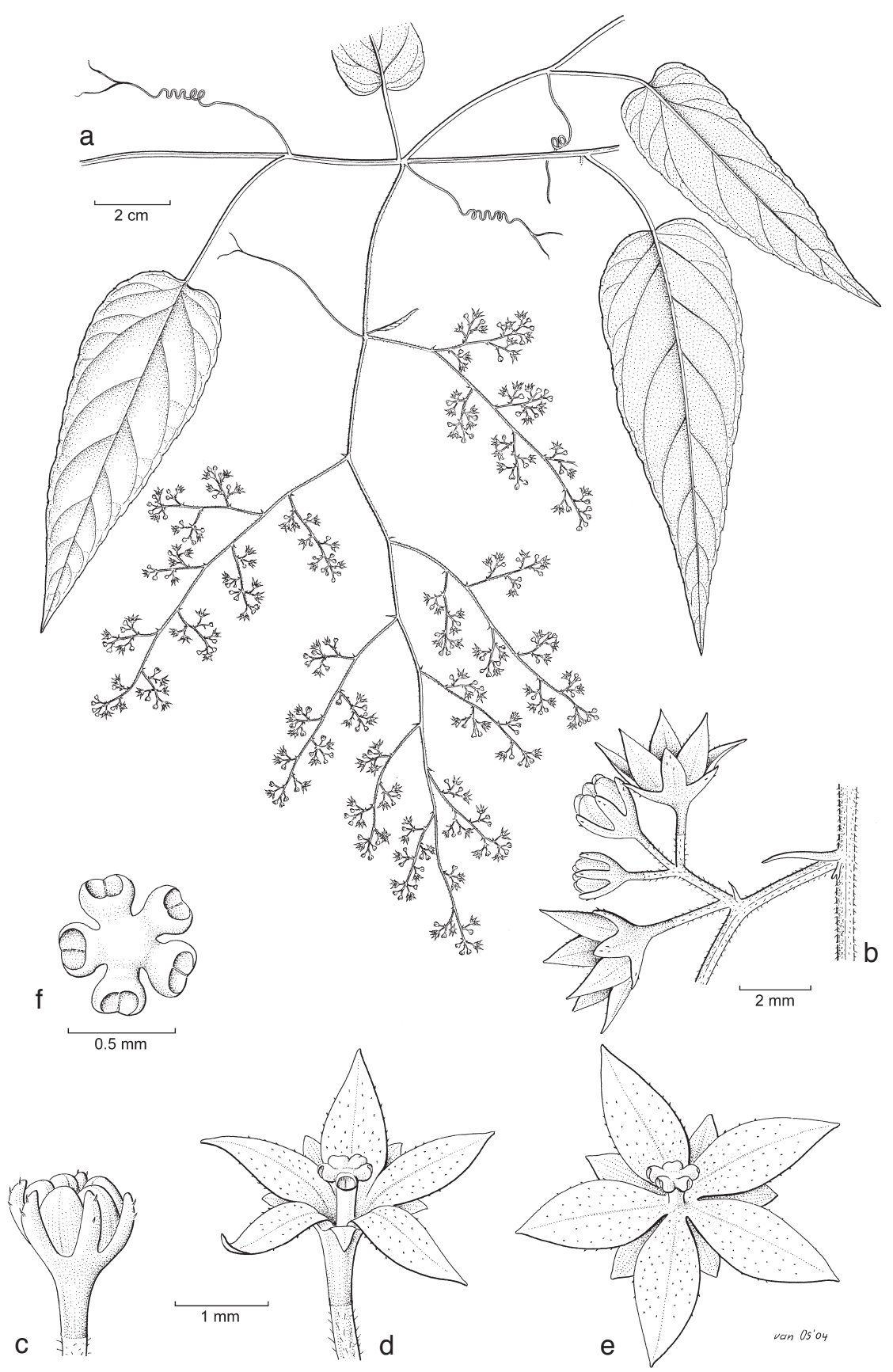

Fig. 2. a: Gynostemma pentaphyllum (Thunb.) Makino forma simplicifolium (Blume) W.J. de Wilde \& Duyfjes. Stem section with male inflorescence. - b-f: Gynostemma pentaphyllum (Thunb.) Makino forma pentaphyllum. b. Male inflorescence; c. male bud; d, e. male flowers; f. androecium seen from above (a: Van Ooststroom 14084; b-f: De Wilde \& Duyfjes 21779). 




Fig. 3. Gynostemma pentaphyllum (Thunb.) Makino forma pentaphyllum. a. Branch section with female inflorescences; b. female inflorescence; c. female flower; d. gynoecium; e. node with infructescence; f. fruit; g. seed (a-d: Kock 8; e-g: Soegandiredja 281). 

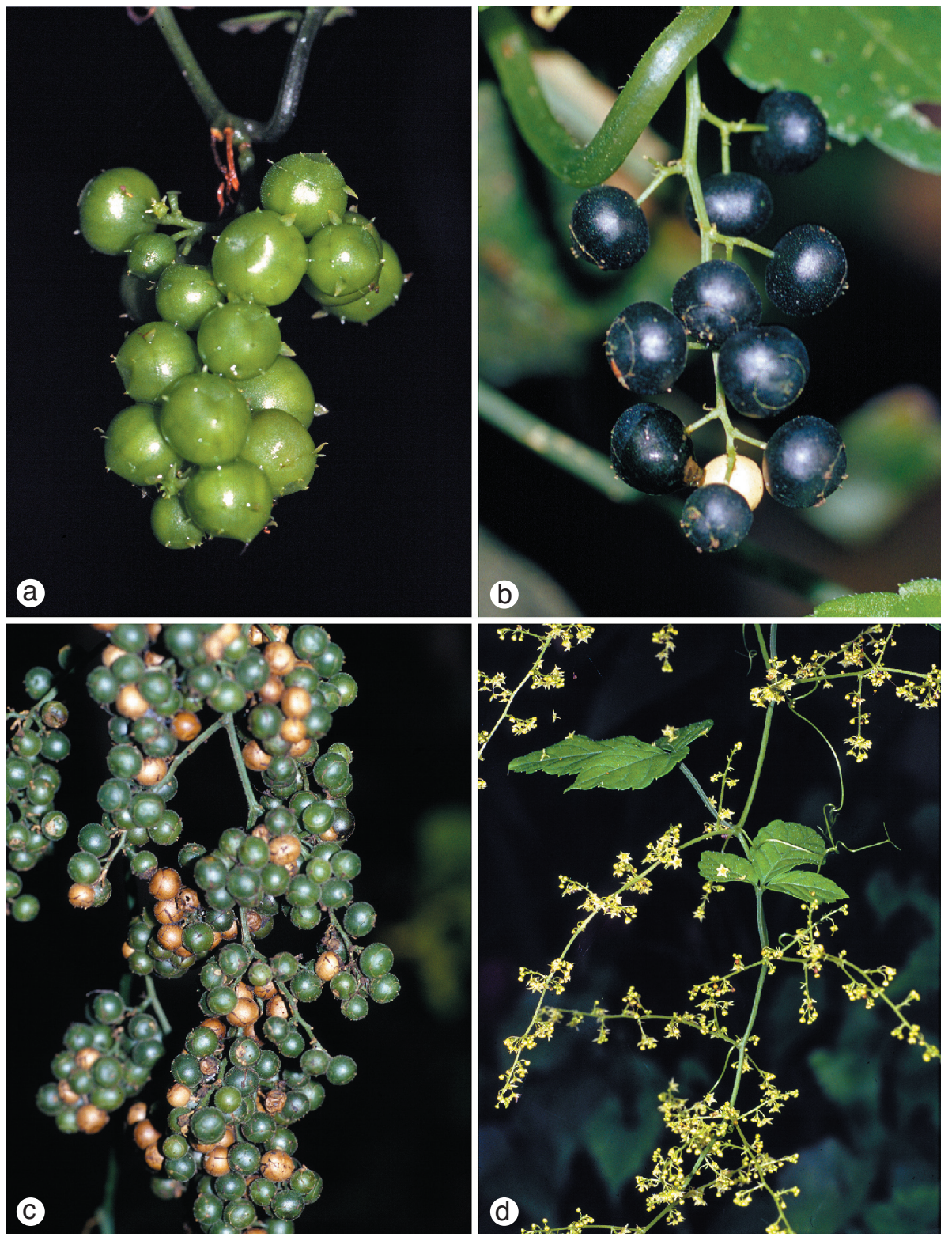

Plate 1. Gynostemma pentaphyllum (Thunb.) Makino: a. forma simplicifolium (Blume) W.J. de Wilde \& Duyfjes; b, d. forma pentaphyllum; c. forma dasycarpum (C.Y. Wu in C.Y. Wu \& S.K. Chen) W.J. de Wilde \& Duyfjes (a: De Wilde et al. SAN 144795, Sabah; b: Koonthunthod et al. 325, Thailand; c: Postar et al. SAN 144099, Sabah; d: De Wilde et al. 21724, Bali). Photos: De Wilde. 


\section{Subgenus TRIROSTELLUM}

Gynostemma Blume subg. Trirostellum (Z.P. Wang \& Q.Z. Xie) C.Y. Wu \& S.K. Chen (1983) 364; S.K. Chen (1986) 273; (1995b) 405. - Trirostellum Z.P. Wang \& Q.Z. Xie (1981) 481. - Type: Trirostellum yixingensis Z.P. Wang \& Q.Z. Xie.

Fruit capsular, opening with 3 valves.

\section{Gynostemma microspermum C.Y. Wu \& S.K. Chen - Fig. 4}

Gynostemma microspermum C.Y. Wu \& S.K. Chen (1983) 364, f. 4 ('microsperma'); S.K. Chen (1986) 274, f. 68: 1-6; (1995a) 392, f. 104: 1-6; (1995b) 406, f. 4: 4. - Type: Li 2805 (KUN, not seen), China (Yunnan).

Perennial creeper or climber, 1-2 m long; tuber $5 \mathrm{~cm}$ diam.; leafy stem 1-2 mm thick, finely sparsely hairy or subglabrous. Leaves: blade (3- or) 5-or 7-foliolate, if 7-foliolate the outer leaflets by far the smallest, leaf blade subcircular in outline, 4-12 cm diam., middle leaflet elliptic-narrowly elliptic or \pm rhomboid, $2.5-8$ by $1.5-3(-4) \mathrm{cm}$, margin dentate-serrate, with 8-12 lateral nerves, raised or not below, lower leaf surface (sub) glabrous, leaf margin and nerves below finely hairy; petiolule 4-8 mm long; petiole 2-4 cm long, (sub)glabrous. Male inflorescences: many-flowered, paniculate, 2 or 3 times branched, $5-12$ by $2-5 \mathrm{~cm}$ (sometimes aggregated in larger panicles), partly finely hairy, flowers in fascicles or short terminal racemes of 3-7 flowers; peduncle 1-2 cm long; bracts minute, c. $0.5 \mathrm{~mm}$ long. Male flowers: pedicel 1.5-2 mm long, articulate at c. $1 / 4$ from the apex; perianth $2.5-3 \mathrm{~mm}$ diam.; receptacle shallow, $0.5-0.7 \mathrm{~mm}$ wide, glabrous, sepals narrowly elliptic, c. $1 \mathrm{~mm}$ long, glabrous; petals ovate to narrowly elliptic and narrowed in the upper half, c. $1.5 \mathrm{~mm}$ long, acute-acuminate, in apical part inside papillose and with c. $0.5 \mathrm{~mm}$ long hairs, especially on or near the margin; staminal column c. $0.4 \mathrm{~mm}$ long, glabrous, synandrium c. $0.4 \mathrm{~mm}$ diam., anthers subglobose, sessile, c. $0.2 \mathrm{~mm}$ diameter. Female flowers: not seen. Infructescences: consisting of numerous fruits in spike-like condensed panicles $3-5$ by $1.5 \mathrm{~cm}$, sometimes aggregated in larger compound infructescences. Capsules: subglobose, 3-4 mm diam., pergamentaceous, glabrous, at apex truncate, 3-valved, opening inwards. Seeds: few, compressed, subcircular or heart-shaped, c. 2 $\mathrm{mm}$ diam., hardly $1 \mathrm{~mm}$ thick, pale brown, faces finely warty, margin flat, wing-like, narrow but distinct.

Distribution - China (Yunnan); Thailand (Doi Chiang Dao, 8 collections seen).

Habitat \& Ecology - Exposed on limestone bed-rock; at 1750-2400 m altitude. Flowering in Thailand: September-November; fruiting: October-December.

Field-notes - Sepals and petals pale yellow-green. Leaflets and fruits yellowgreen.

Notes - 1. In Flora Yunnanica (Chen, 1995a: f. 104: 2, 3) the conspicuous hairs on the petals of male and female flowers are not drawn, but otherwise the figure agrees with the Thai material. In Yunnan the species occurs between 800 and 1350 metres.

2. The chromosome number is: $2 \mathrm{n}=22$ (Gao et al., 1995). 


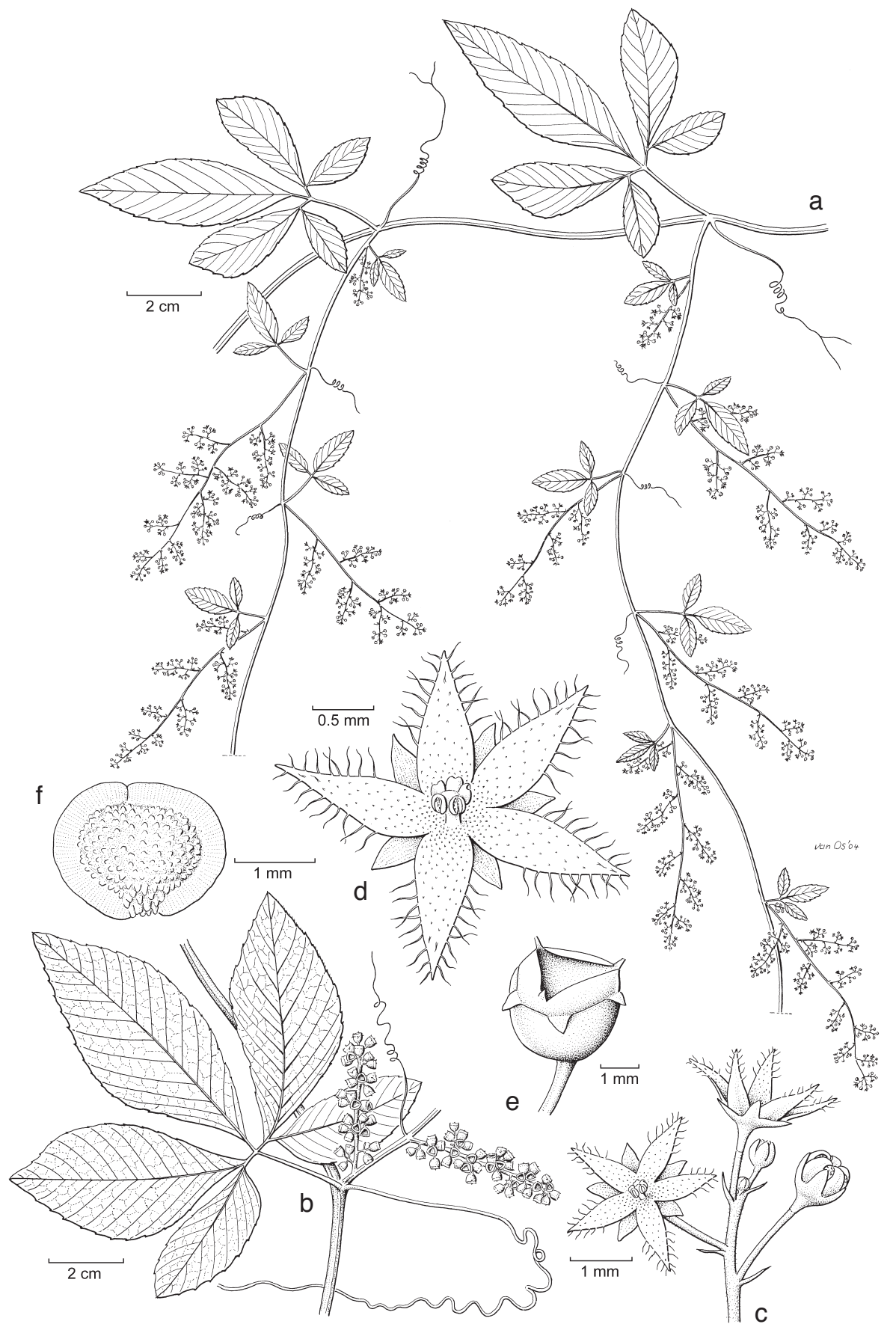

Fig. 4. Gynostemma microspermum C.Y. Wu \& S.K. Chen. a. Twig section with male inflorescences; b. habit of infructescences; c. detail of male inflorescence; d. male flower; e. fruit, dehiscent by 3 valves; f. seed (a-d: Murata, Iwatsuki \& Phengklai T-15118; e, f: Maxwell 95-1092). 


\section{ACKNOWLEDGEMENTS}

We are grateful to Dr. Kongkanda Chayamarit and the staff of BKF and Dr. Jamili Nais and his staff for facilitating collection of Gynostemma in the wild, respectively in Thailand and Sabah, in the Kinabalu National Park. Herbarium collections from BK, BKF, BM, BO, BRI, BRUN, CMU, E, K, L, MO, P, QBG, SAN, SING, SNP, TCD, and W were used for the present treatment of Gynostemma. We thank Erna Shinta (CIMTROP Herbarium, Palankaraya University, Indonesia) and Phornpitak Panyarat (BKF) who carried out a useful study of G. pentaphyllum formae simplicifolium and pentaphyllum for Java during the Bogor Training Course in 1996, sponsored by ARCBC (Asean Regional Center for Biodiversity Conservation, Manila, a joint co-operation project between ASEAN and the European Union). Dr. Dan Nicolson (US) provided information on literature. As usual Dr. J.F. Veldkamp (Leiden) kindly provided the translations into Latin of the diagnoses of the new taxa, Jan van Os (Leiden) prepared the beautiful drawings, Ben Kieft (Leiden) scanned the drawings, and Luc Willemse (Leiden) helped with the production of the exsiccata list, using BRAHMS.

\section{REFERENCES}

Arnott, G.A.W. 1836. Pugillus plantarum Indiae Orientalis. Nov. Actorum Acad. Caes. Leop.-Carol. Nat. Cur. 18, 1: 319-356a-d.

Backer, C.A. 1964. Cucurbitaceae. In: C.A. Backer \& R.C. Bakhuizen van den Brink Jr., Flora of Java 1: 292-307. Noordhoff, Groningen.

Blume, C.L. 1825. Bijdragen tot de Flora van Nederlandsch Indië 1: 23-24. Ter Lands Drukkerij, Batavia.

Chakravarty, H.L. 1946. Studies on Indian Cucurbitaceae; with special remarks on distribution and uses of economic species. Indian J. Agric. Sci. 16: 1-90.

Chakravarty, H.L. 1959. Monograph on Indian Cucurbitaceae. Rec. Bot. Surv. India 17: 1-234.

Chen, S.K. 1986. Gynostemma. In: A.M. Lu \& S.K. Chen (eds.), Fl. Reipubl. Popularis Sin. 73: 265-277. Science Press, Beijing.

Chen, S.K. 1995a. Gynostemma. In: C.Y. Wu, C. Chen \& S.K. Chen (eds.), Flora Yunnanica 6: 384-392. Science Press, Beijing.

Chen, S.K. 1995b. A classificatory system and geographical distribution of the genus Gynostemma Bl. (Cucurbitaceae). Acta Phytotax. Sin. 33: 403-410.

Clarke, C.B. 1879. Cucurbitaceae. In: J.D. Hooker, Flora of British India 2: 604-635. Reeve \& Co., London.

Cogniaux, C.A. 1881. Cucurbitaceae. In: A. \& C. de Candolle, Monographiae Phanerogamarum Prodromi 3: 325-951.

Craib, W.G. 1918. Contributions to the Flora of Siam. Additamentum 10. Kew Bull.: 362-363.

Craib, W.G. 1931. Cucurbitaceae. Flora Siamensis Enumeratio 1: 750-770. Siam Society, Bangkok.

De Wilde, W.J.J.O. \& B.E.E. Duyfjes. 2003. Revision of Neoalsomitra (Cucurbitaceae). Blumea 48: $99-121$.

Ding, J.N. 1990. A new variety of Gynostemma yixingense. Bull. Bot. Res., Harbin 10: 71-72.

Gagnepain, F. 1921. Cucurbitacées. Flore Générale de l'Indo-Chine 2: 1030-1095. Masson \& Cie., Paris.

Gao, X.F., S.K. Chen, Z.J. Gu \& J.Z. Zhao. 1995. A chromosomal study on the genus Gynostemma (Cucurbitacae). Acta Bot. Yunnan. 17: 312-316.

Griffith, W. 1854. Some account of the botanical collection, brought from the eastward, in 1841, by Dr. Cantor. J. Asiat. Soc. Bengal 23: 623-650, pl. 1-4.

Hô, P.H. 1991. An illustrated Flora of Vietnam 1, 2. Mekong printing, Santa Ana.

Hooker, J.D. 1867. Cucurbitaceae. In: G. Bentham \& J.D. Hooker, Genera plantarum 1. Reeve \& Co., London.

How, F.C. \& C.C. Chang. 1964. Cucurbitaceae. In: W.Y. Chun, C.C. Chang \& F.H. Chen (eds.), Flora Hainanica 1: 468-485. Science Press, Beijing. 
Jeffrey, C. 1980. The Cucurbitaceae of Eastern Asia. Roy. Bot. Gard., Kew.

Keraudren-Aymonin, M. 1975. Cucurbitacées. In: A. Aubréville \& J.-F. Leroy (eds.), Flore du Cambodge, du Laos et du Viêt-nam 15: 1-114. Mus. Nat. d'Hist. Nat. Paris.

Lauener, L.A. 1972. Catalogue of the names published by Hector Léveillé: VII. Notes Roy. Bot. Gard. Edinburgh 31: 429-432.

Léveillé, A.A.H. 1909. Decades plantarum novarum. Repert. Spec. Nov. Regni Veg. 7: 337-340.

Léveillé, A.A.H. 1912a. Decades plantarum novarum. Repert. Spec. Nov. Regni Veg. 10: 348-352.

Léveillé, A.A.H. 1912b. Decades plantarum novarum. Repert. Spec. Nov. Regni Veg. 11: 295-307.

Léveillé, A.A.H. \& E. Vaniot. 1905. Bull. Soc. Agric. Sci. Arts Sarthe: 41 (not seen).

Makino, T. 1902. Observations on the Flora of Japan. Bot. Mag. (Tokyo) 16: 179.

Merrill, E.M. 1938. New or noteworthy Indo-Chinese plants. J. Arnold Arbor. 19: 69-70.

Miquel, F.A.G. 1856. Flora Indiae Batavae 1, 1: 652-683. Van der Post, Amsterdam.

Moritzi, A. 1846. Systematisches Verzeichniss der von H. Zollinger in den Jahren 1842-1844 auf Java gesammelten Pflanzen. Druck von F.X. Zepfel.

Müller, E.G.O. \& F.A. Pax. 1889. Cucurbitaceae. In: A. Engler \& K. Prantl, Die natürlichen Pflanzenfamilien 4, 5: 1-39. Engelmann, Leipzig.

Ohwi, J. 1965. Flora of Japan. Smithsonian Institution, Washington, D.C.

Ridley, H.N. 1920. On a collection of plants from Peninsular Siam. J. Fed. Malay States Mus. 10: 93.

Roemer, M.J. 1846. Peponiferarum. Synopses Monographicae 2. Landes-Industrie-Comptoirs, Weimar.

Thunberg, C.P. 1784. Fl. Japonica. Leipzig.

Thwaites, G.H.K. 1859. Enumeratio Plantarum Zeylaniae 2. Dulau \& Co., London.

Van Royen, P. 1982. The Alpine Flora of New Guinea 3. Cramer, Vaduz.

Wallich, N. 1831. Plantae Asiaticae rariores 2. Treuttel \& Würtz, London.

Wang, Z.P. \& Q.Z. Xie. 1981. Trirostellum, a new genus of the Cucurbitaceae from China. Acta Phytotax. Sin. 19: 481-484.

Winkler, H. 1912. Beiträge zur Kenntnis der Flora und Pflanzengeographie von Borneo. II. Bot. Jahrb. Syst. 48: 118.

Wu, C.Y. \& S.K. Chen. 1983. A study of the genus Gynostemma Bl. (Cucurbitaceae) from China. Acta Phytotax. Sin. 21: 355-369.

\section{IDENTIFICATION LIST}

$1=$ Gynostemma intermedium

2 = Gynostemma papиапит

$3=$ Gynostemma pentaphyllum s.l.

$3 \mathrm{a}=$ Gynostemma pentaphyllum forma dasycarpum

$3 \mathrm{~b}=$ Gynostemma pentaphyllum forma fasciculare

$3 \mathrm{c}=$ Gynostemma pentaphyllum forma grandiflorum

$3 \mathrm{~d}=$ Gynostemma pentaphyllum forma knemandrum

$3 \mathrm{e}=$ Gynostemma pentaphyllum forma pentaphyllum

$3 \mathrm{f}=$ Gynostemma pentaphyllum forma pubescens

$3 \mathrm{~g}=$ Gynostemma pentaphyllum forma simplicifolium

$4=$ Gynostemma microspermum

Alcasid PNH 1690: 3e - Alston 14669: 3e - Anderson 4620: 3g; 5285: 3; S 26073: $3 \mathrm{a}$ - Anusarnsunthorn 43: 3e - Arens 76: 3e - Awa S 45288: 3a; S 46717: 3a.

Backer 36989: 3g; 37304: 3e - Bakhuizen van den Brink Jr. 365: 3e; 601: 3e; 1673: 3e - Bakhuizen van den Brink Sr. 2855: 3e; 4558: 3e; 4714: 3e; 4775: 3g - Barbon PPI 13148: 3e - Barnes 619:3 - Bartlett 302:3e - Beaman 7217: 3a - Beccari 27 A: 3e; 805: 3e - Bicknell 1068: 3e; 1642: 3e - Bloembergen 614: 3e; 4420: 3e - Blume 1429: 3e; 1493: $3 \mathrm{~g}-$ Bor 15915: 3; 15928: 3; 16334: 3 - Borgmann 181: 2 - Boufford 24141:3; 24372: 3 - Brass 22831: 2 - Bubung SK 223: 3e - Bünnemeijer 2720: 3e; 3142: 3e; 3421: 3e. 
Carr 13960: 3e - Chai S 36046: 3a - Chand 3923: 3 - Chew CWL 326: 3a; CWL 1299: 3a - Chow 76107: 3 - Clemens 27488: 3a; 27941: 3a; 29237: 3g; 29790: 3a; 30680: 3g; 40280: 3g - Coert 571: 3e - Conklin PNH 19131: 3e; PNH 80525: 3e - Coode NGF 32683: 1; NGF 32913: 2; NGF 40286: 2 - Craib 328: 3.

d'Alleizette 2684: 3e - Danser 5818: 3g; 6098: 3g - Darbyshire 246: 3e - De Vogel 787: 3a; 859: 3a; 1785: 3a; 5164: 3b; 7035: 3b - De Wilde 12497: 3e; 19246: 3e; 21700: 3e; 21702: 3e; 21714: 3e; 21724: 3e; 21725: 3e; 21768: 3g; 21769: 3e; 21779: 3e; 21798: 3e; 21842: 3g; 21880: 3e; 21890: 3e; 21958: 3g; 21980: 3g; 22053: 3g; 22054: 3a; 22126: 3e; 22136: 3e; 22137: 3f; 22144: 3c; 22172: 3e; 22173: 3e; 22174: 3e; 22277: 3g; 22282: 3e; 22286: 3e; SAN 139457: 3g; SAN 139458: 3g; SAN 143926: 3a; SAN 143954: 3a; SAN 143969: 3a; SAN 143999: 3a; SAN 144012: 3g; SAN 144015: 3g; SAN 144021: 3a; SAN 144024: 3a; SAN 144536: 3g; SAN 144539: 3a; SAN 144795: 3g; SAN 144796: 3g - Den Hoed 918: 3e - Dickason 9136: 3 - Dorgelo Ngebel 18: 3e.

Edaño PNH 7233: 3e; PNH 11077: 3e; PNH 11077A: 3e; PNH 37122: 3e; PNH 37757: 3e; PNH 38529: 3e - Elmer 9485: 3e; 15304: 3e; 18302: 3e - Endert 5226: 3a - Eyma 1010: 3b.

Floyd \& Womersley 6779: 1 - Fukuoka T 4367: 3e; T 62173: 3; T 63709: 3e.

Gaerlan PPI 10596: 3e; PPI 10864: 3e; PPI 19849: 3e; PPI 20363: 3e; PPI 26169: 3g; PPI 26635: 3e; PPI 26636: 3e - Garcia PPI 22968: 3e - Gardner 9536: 3e - Garrett 190: 3d; 320: 3e; 520: 3e; 875: 3e - Geesink 8015: 3c; 8138: 3e - George S 38268: 3a - Gibot SAN 94561: 3a - Gilli 484: 2 - Gravendeel BG 557: 3e; BG 558: 3e - Griffith 762: 3e; 2524: 3; 2525: 3.

Hansen 12166: 3e - Hatusima 19088: 3 - Herb. Hort. Bot. Bog. 272: 3e - Hoogland 7540: 2- Hu 22078: 3 .

Iboet 250 A: $3 \mathrm{e}-$ Iwatsuki T 268: 3.

Jacobs 4819: 3e - Jermy 14233: 3g - Jeswiet 38: 3e - Johansson, Nybom \& Riebe 384: $3 \mathrm{~b}$.

Kao 9940: 3 - Kato 1139: 3e; C 7100: 3e - Kerr 747: 3e; 1434: 3e; 16901: 3e; 18414: 3e; 21248: 3e - Kloss 6901: 3e - Kock 8: 3e; 9: 3e; 10: 3e - Koelz 26192a: 3; 30719: 3; 31074: 3 - Kokawa 3021: 3g; 4701: $3 \mathrm{~g}$ - Koonthunthod 325: 3e; 331: 3e; 332: 3e - Koorders 15395: 3e; 15905: 3b; 15910: 3b; 15919: 3b; 20363: 3e; 25938: 3g; 37320: 3e; 42145: 3g; 43254: 3e; 43456: 3e - Koster BW 13743: 3e - Kostermans 411: 3e; 711A: 3e; 847: 3e; 1081: 3e; 1646: 3e - Koyama T 33874: 3 - Kramer 7601: 3 - Krispinus SAN 88225: 3a; SAN 121821: 3a.

Lam 3625: 3e - Larsen 711: 3e; 2557: 3d; 3092: 3e; 46569: 3f; 46776: 3c - Ledermann 10268: 3 - Lee S 40031: 3a - Lörzing 5842: 3e; 11339: 3e.

Madulid PPI 27400: 3e - Mai Van Xinh MVX 358: 3e - Maikin SAN 132157: 3a - Mamit S 37422: 3a - Martin \& Bodinier 1791: 3 - Maxwell 76-479: 3e; 87-772: 3e; 87-820: 3e; 891116: 3e; 89-1286: 3d; 89-1357: 3e; 89-1368: 3e; 90-935: 3f; 90-1147: 3f; 91-713: 3f; 91-719: 3e; 92-524: 3e; 93-974: 3e; 93-1121: 3e; 93-1122: 3e; 94-982: 3e; 94-1001: 3c; 95-524: 3e; 951092: 4; 96-835: 3e; 96-890: 3e; 96-1029: 3e; 96-1132: 3e; 97-823: 3e; 97-891: 3f; 97-932: 3e; 98-700: 3f; 99-184: 3e; 00-337: 3e - Meijer 81: 3e; 5328: 3e; 5626: 3e; 7259: 3e - Mendoza PNH 18297: 3e; PNH 97486: 3e - Merrill BS 1740: 3e; BS 7662: 3e - Middleton 146: 3e; 554: 3e; 1053: 3; 1678: 3e; 1833: 3e - Mousset 134: 3e - Murata T 14865: 3e; T 15118: 4; T 16918: 3e; T 38547: 3; T 51479: 3.

Natti V 1: 4 - Nicolson 2413: 3 - Nooteboom 5260: 3e - Nur SF 32710: 3g.

Paie S 36276: 3 g - Partomihardjo 1500: 3e - Petrmitr 73: 3e - Phengklai 2930: 3e; 11417: 3 — Phonsena 3519: 3e; 3918: 3e; 3928: 3e; 4437: 3c; 4438: 3c; 4478: 3d; 4682: 3e; 4683: 3e; 4689: 3f; 4702: 3e - Phusomsaeng 7A: 3e - Plernchit 494: 3 - Poilane 11212: 3f; 26701: 3e - Postar SAN 144099: 3a; SAN 144252: $3 \mathrm{~g}$ - Proefstation voor de Javasuikerindustrie 8635: 3e - Pruesapan KP 36: 3e - Pullen 422: 1.

Raap 919: 3g - Ramos BS 38643: 3e - Reynoso PPI 3518: 3e; PPI 3615: 3e; PPI 21179: 3e; PPI 21778: 3e; PPI 24369: 3e; PPI 27207: 3e - Robinson BS 9315: 3e.

Sangkhachand 1426: 3e; 40993: 3 - Santisuk 201: 3 - Santos BS 31941: 3e - Sayers NGF 19787: 1 - Schiffner 2718: 3e; 2719: 3e - Schodde 1882: 2 - Shimizu T 9066: 3; T 10037: 4; T 11624: 3a; T 18488: 3; T 18489: 3; T 20928: 4; T 21619: 3 - Sibat ak Luang S 22371: 3a - Sino-American Guizhou Botanical Expedition 1986 123: 3; 1892: 3 - Smitinand 734: 3; 1660: 3e; 6251: 4; 8747: 3e - Soegandiredja 281: 3e - Soejarto 9757: 3e; 10418: 3e; 11692: 3e - Soetisna 
27: 3a - Soradet 39: 3f - Steiner PNH 39643: 3e - Stevens LAE 55693: 2 - Suddee 2462: 4; 2499: 4; 2510 A: 3e; 2510B: 4; 2511: 3e; 2515: 4 - Sulit PNH 14510: 3e - Surbeck 221: 3e - Sørensen 628: 3; 3669: 3.

Tagawa T 924: 3e; T 1938: 3e - Takahashi T 63558: 3 - Takeuchi 20140: 2 - Taquet 2744: 3e; 2745: 3e - Thorel 3468: 3f - Tosari 68: 3e - Timothy NGF 27980: 3e - Tsang 29196: 3e; 29224: 3e.

University of San Carlos 837: 3e - Unknown 19b: 3e.

Van Beusekom 3779: 3f; 4184: 3 - Van Ooststroom 14084: 3g; 14108: 3e - Van Royen 11361: 2; NGF 17541: 1 - Van Steenis 2359: 3e; 2379: 3e; 3235: 3e; 6439: 3e; 18298: 3e; 20848: 3 - Veldkamp 5888: 2; 6263: 2 - Vink 16151: 2; 17364: 2.

Wallich 3726: 3e; 3727: 3e - Winkler 1080: 3a; 2757: 3a - Womersley \& Sleumer NGF 13999: 1 - Wong WKM 2357: 3a - Wood A 3991: 3a.

Yates 1924: 3e. 